\title{
ANÁLISIS SEMIPARAMETRICO DE LOS FACTORES ASOCIADOS A LA SOSTENIBILIDAD DE LOS EMPRENDIMIENTOS*
}

\author{
WALTHER REINA GUTIÉRREZ**, CLAUDIA INÉS SEPÚLVEDA RIVILLAS**** \\ \& GABRIEL JAIME GONZÁLEZ URIBE******* \\ UNIVERSIDAD DE ANTIOQUIA
}

Recibido/ Received/ Recebido: 31/10/2016 - Aceptado/ Accepted / Aprovado: 05/12/2016

\begin{abstract}
Resumen
El presente artículo, producto de una investigación, pretende identificar y analizar los factores que determinan la sostenibilidad de los emprendimientos, evidenciando la relevancia de los efectos individuales y cruzados, relacionados con el emprendedor, el emprendimiento y el entorno, en la Subregión del Bajo Cauca - Colombia, para lo cual se abordó una metodología cuantitativa mediante un modelo semiparamétrico. Se utilizó una muestra de 101 emprendedores, 73 activos y 28 inactivos. El principal aporte de la investigación es determinar el grado en que cada variable, individualmente y en interacción con otras, afecta la supervivencia de los emprendimientos. Las variables que inciden sobre la supervivencia son: elaboración del plan de negocios, ubicación, endeudamiento, diversificación de la oferta y forma jurídica.

Palabras clave: Emprendimiento; Supervivencia; Sostenibilidad; Análisis Semiparamétrico.
\end{abstract}

\section{SEMIPARAMETRIC ANALYSIS OF FACTORS ASSOCIATED TO ENTERPRISES SUSTAINABILITY}

\begin{abstract}
This article, a research product, aims to identify and analyze the factors that determine the sustainability of the enterpreneurships, evidencing the relevance of the individual and crossed effects, related to the entrepreneur, entrepreneurship and the environment, in the Subregion of Bajo
\end{abstract}

\footnotetext{
El presente artículo es derivado del proyecto de investigación: Factores determinantes en la sostenibilidad de los emprendimientos en el tiempo en la Subregión del Bajo Cauca. Aprobado y financiado en la Convocatoria de Proyectos de Investigación- Regionalización 2013 del Comité para el Desarrollo de la Investigación (CODI) de la Universidad de Antioquia.

* Economista de la Universidad Autónoma Latinoamérica, Especialista en Finanzas, Preparación y Evaluación de Proyecto de la Universidad de Antioquia, Magister en Finanzas de la Universidad EAFIT. Profesor de la Universidad de Antioquia, Facultad de Ciencias Económicas, Departamento de Ciencias Administrativas, adscrito al Grupo de Investigación en Finanzas GIFi. Correo electrónico: walter.reina@udea.edu.co. Teléfono 2195853. Dirección postal: calle 67 No. 53 - 108 bloque 13, oficina 410.

*** Administradora de Empresas de la Universidad de Antioquia, Especialista en Finanzas, Preparación y Evaluación de Proyectos de la Universidad de Antioquia, Magíster en Finanzas de la Universidad Eafit y candidata a Doctora en Dirección de Empresas de la Universidad de Valencia. Profesora de la Universidad de Antioquia, Facultad de Ciencias Económicas, Departamento de Ciencias Administrativas, adscrita al Grupo de Investigación en Finanzas GIFi. Correo electrónico: claudia.sepulveda@udea.edu.co. Teléfono 2195830. Dirección postal: Carrera 39 No. 47-53 Apto 1102, Medellín.

Economista de la Universidad de Antioquia, Magister en Economía de la Universidad de Antioquia. Profesor del Instituto Tecnológico Metropolitano ITM, Facultad de Ciencias Económicas y Administrativas. Adscrito al grupo de investigación de Ciencias Administrativas del ITM y al grupo de investigación de Macroeconomía Aplicada de la Universidad de Antioquia. Correo electrónico: elgabotb@gmail.com, Teléfono 4600727 Ext 5622. Dirección postal Calle 54 A \#30-01, bloque L oficina 405.
} 
Cauca - Colombia, for which a quantitative methodology was addressed through a semi-parametric model. We used a sample of 101 entrepreneurs, 73 active and 28 inactive. The main contribution of this research is to determine the degree to which each variable, of those identified for the study -elaboration of the business plan, location, indebtedness, diversification of the offer and legal form, individually and in interaction with others, affects the survival of the enterpreneurships.

Keywords: Entrepreneurship; Survival; Sustainability Semi-parametric Analysis.

\title{
ANÁLISE SEMIPARAMÉTRICO DOS FATORES ASSOCIADOS À SUSTENTABILIDADE DOS EMPREENDIMENTOS
}

\begin{abstract}
Resumo
O presente artigo, produto de pesquisa, pretende identificar e analisar os fatores que determinam a sustentabilidade dos empreendimentos, evidenciando a relevância dos efeitos individuais e cruzados, relacionados com o empreendedor, o empreendimento e o meio, na subregião do Baixo Cauca - Colômbia, para o qual se abordou uma metodologia quantitativa mediante um modelo semiparamétrico. Utilizou-se uma mostra de 101 empreendedores, 73 ativos e 28 inativos. A principal contribuição desta pesquisa é determinar o grau em que a cada variável, das identificadas para o estudo - elaboração do plano de negócios, localização, endividamento, diversificação da oferta e forma jurídica -, individualmente e em interação com outras, afeta a sobrevivência dos empreendimentos.
\end{abstract}

Palavras chave: Empreendimento; Sobrevivência; Sustentabilidade; Análise semiparamétrico

Reina, W., Sepúlveda, C. \& González, G. (2018) Análisis semiparametrico de los factores asociados a la sostenibilidad de los emprendimientos. En: Revista de la Facultad de Ciencias Económica: Investigación y Reflexión. rev.fac.cienc.econ, XXVI (1), DOI: https://doi. org/10.18359/rfce.3144

JEL: M13, $C 14$.

\section{Introducción}

Como estrategia mundial, para la reducción del desempleo ${ }^{1}$ y el jalonamiento de la economía, entre otras variables, es evidente el fortalecimiento del emprendimiento, tema en el cual Antioquia ha sido uno de los departamentos más destacados en Colombia, gracias al compromiso de sus gobernantes y de los diferentes actores de la economía, tanto a nivel privado como público y a nivel de empresas del sector real y de las instituciones educativas.

La tendencia anterior tiene mucho sentido, pues como lo plantea Global Entrepreneurship Monitor - GEM, uno de los principales inductores para

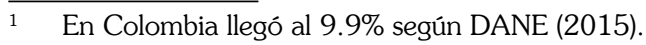

generar innovación permanente es fortaleciendo el ecosistema del emprendimiento y lograr crear y sostener emprendimientos cuyos niveles de innovación garanticen aportes significativos en el crecimiento de la economía, los cuales según Pereira \& Medina (2012), difieren de país en país debido a la dinámica emprendedora.

Producto de ese impulso al emprendimiento el número de unidades productivas creadas, principalmente Micro, Pequeñas y Medianas - Mipymes, representa un porcentaje muy representativo en todas las economías, que en el caso colombiano ha llegado a un $96 \%$ del total de las empresas del país y a un $99 \%$ de Departamento de Antioquia, lo cual da cuenta del papel que esta categoría de empresas juega al interior del país, aportando crecimiento de la economía y generando nuevas fuentes de em- 
pleo. Pese a esto, y al gran número de empresas que se constituyen a diario, las microempresas reportan una alta tasa de mortalidad durante sus primeros años de vida.

En línea con lo anterior, un análisis de supervivencia realizado a las empresas creadas en el período 2000-2012, en Antioquia-Colombia, permite evidenciar que algunos de los factores que influyen en la sostenibilidad son el bajo perfil del emprendedor, la insuficiente planeación estratégica y de mercado, la alta competencia en el mercado, la deficiente planificación financiera y la baja generación de valor agregado, es así como la estadística muestra que después de tres años de creación el $26,73 \%$ de las empresas fueron cerradas, después de siete años el $40,41 \%$ y doce años después había desaparecido el $48,14 \%$. Además, se encontró evidencia significativa de la influencia del tamaño y el sector económico en la sostenibilidad de las empresas (Cámara de Comercio de Medellín para Antioquia, 2013)

De acuerdo a los anteriores planteamientos, el objetivo de este artículo ${ }^{2}$ es realizar un análisis, a través de un modelo semiparamétrico, de los factores que determinan la sostenibilidad de los emprendimientos, evidenciando los efectos individuales y cruzados y la relevancia de los mismos. Estos factores son clasificados en: factores internos, relacionados con las características de la empresa, factores del emprendedor referentes a sus habilidades, rasgos $y$ competencias, $y$ factores del entorno que tienen que ver con las características del ecosistema donde opera la empresa.

El presente documento se estructura en cinco partes, incluyendo esta introducción. En la segunda parte se presenta la revisión de la literatura, seguidamente se plantea la metodología, posteriormente

2 El presente artículo es una continuación del artículo publicado por Sepulveda \& Reina (2015), titulado Sostenibilidad de los emprendimientos: Un análisis de los factores determinantes, en el cual a través de un modelo no paramétrico como el Kaplan - Meier, usando curvas de supervivencia, se identificaron y analizaron los factores que insidian en la sostenibilidad de los emprendimientos. los resultados y se finaliza con la presentación de las conclusiones.

\section{Revisión de la literatura}

\subsection{Sostenibilidad y emprendimiento sostenible}

La sostenibilidad hace referencia a la capacidad de un emprendimiento de mantenerse en el tiempo, en la medida que se desarrollan capacidades y competencias. En este caso específico, se refiere a la capacidad de una organización de mantener un posicionamiento en el mercado y en el sector, que le permita sobrevivir a través de los años, generando rentabilidad y valor, tanto para la propia empresa, como para el sector en que se localiza y para los empleados (Schaltegger \& Wagner, 2011).

Rajasekaran (2013) asocia la sostenibilidad a la necesidad primordial de encontrar un balance entre la sociedad, la economía y la ecología, para poder lograr un futuro desarrollo de la sociedad y a gran escala de la humanidad. El emprendimiento sostenible es un compromiso permanente de la empresa, para que sus procesos funcionen de manera ética y contribuyan al desarrollo económico, al mismo tiempo que se mejora la calidad de vida de los empleados y de la comunidad en general, teniendo siempre en cuenta el avance en el tiempo y las futuras generaciones.

Las posturas anteriores, relacionan dos tipos de emprendimientos: sociales y de negocios, cuya sostenibilidad no se ve afectada por la naturaleza de estos, pero si por diversos factores que difieren de acuerdo al tipo de sociedad (Simón, Revuelto \& Medina, 2012), y coinciden en la necesidad de crear valor para todos los stakeholders, como estrategia para garantizar la sostenibilidad.

Por tanto, el emprendimiento sostenible es un concepto multidimensional que se extiende más allá de la protección ambiental, el desarrollo económico y la equidad social. Promover la sostenibilidad del espíritu empresarial significa reconocer y aplicar análisis empresariales que identifiquen oportunidades para aumentar la vida de las empresas que son una fuente positiva de crecimiento económico. La activi- 
dad empresarial solo puede considerarse sostenible una vez que se planifica a largo plazo con el objetivo de obtener ganancias económicas (Yeasmin, 2016)

\subsection{Enfoques del Emprendimiento}

\subsubsection{El enfoque microeconómico}

Explica el papel del emprendedor dentro del entorno económico, mostrando las variables que el individuo tiene en cuenta para tomar la decisión de crear o no empresa, y el impacto que estas decisiones generan en la economía. En este sentido para Baumol (1996) los emprendedores son ingeniosos, creativos, caracterizados por mantenerse en la búsqueda de formas que les generen poder, riqueza y prestigio, y su comportamiento cambia de acuerdo a la economía en que se encuentren. Por su parte Casson (1983) afirma que la decisión de convertirse en empresario, se dará después de haber evaluado la recompensa de esta actividad con el beneficio esperado de la mejor utilización de su tiempo. Paralelamente, Campbell (1992) plantea un modelo de decisión para actos empresariales, en el cual, cualquier acto empresarial estará orientado a aumentar la riqueza y el bienestar del empresario, y por ende la decisión de convertirse en empresario o en empleado, se tomará después de evaluar los beneficios de ambas opciones.

\subsubsection{El enfoque de los rasgos de personalidad}

Analiza al emprendedor desde lo que es, para así poder comprender cómo éstas características influyen en el espíritu empresarial. Así, para Gartner (1988, pp. 21-23) "las características de la personalidad del empresario son auxiliares a los comportamientos del empresario". Por su parte, Frese et al. (1997) afirman que los emprendedores se diferencian por su iniciativa personal, lo cual es un rasgo esencial de las personas que crean su propia empresa.

Iturriaga, Baniandrés \& Eizaguirre (2016) señalan que todos los estudios iniciales sobre el emprendimiento y sus causas, o factores que lo facilitan o lo impiden, se han centrado en el análisis de las características personales del emprendedor, y que aspectos como la personalidad del líder, caracte- rísticas psicológicas y perfil sociocultural han sido identificados como la razón principal que explica el emprendimiento, dando lugar al denominado enfoque de rasgos de personalidad.

Según Moriano, Trejo \& Palací (2001) los emprendedores son portadores de competencias como: adaptabilidad, autonomía, capacidad de asumir riesgos, innovación, proactividad y tolerancia a la incertidumbre.

Ante la afirmación "el emprendedor nace, no se hace”, Wompner (2008, pp. 9-10) señala que, aunque existen individuos que nacen con algunas características favorables para emprender; las instituciones de educación juegan un papel importe en la orientación y el desarrollo de habilidades en los individuos, ayudándoles a promover y desarrollar su espíritu empresarial.

Otro elemento a tener en cuenta es que el emprendimiento es situacional, es decir, aunque existan ciertos rasgos personales de partida, solo en determinadas situaciones las personas deciden emprender. Dado que las circunstancias son importantes, al hablar de emprendimiento es necesario analizar otras variables que definen el entorno, es decir las variables o factores estructurales (Iturriaga, Baniandrés \& Eizaguirre, 2016)

\subsubsection{El enfoque del Comportamiento}

Parte de lo sugerido por Gartner (1988), quien indica que las investigaciones sobre el emprendedor deben realizarse con base en lo que hace y no en lo que es. En este sentido, Iturriaga, Baniandrés \& Eizaguirre (2016) señalan que en este nuevo enfoque no se ha encontrado nada esclarecedor en los rasgos personales de los emprendedores y que lo diferencial entre emprendedores y no emprendedores es lo que hacen y no lo que son. Admitiendo que existen algunas diferencias de personalidad entre personas emprendedoras y no emprendedoras, lo cual no es razón suficiente para emprender

Paralelamente, Lau \& Chan (1994) proponen el método incidente, como alternativa para evaluar los comportamientos del emprendedor; dicho método 
se basa en una metodología de encuestas y estudios de caso. Además, Rodríguez (2009) identifica lo que es el emprendedor - características y personalidad emprendedora - como la base del comportamiento emprendedor, definiendo esto como un enfoque contextual, resultado de múltiples influencias.

\subsubsection{El enfoque Cognitivo}

Presenta la importancia de evaluar el espíritu emprendedor desde lo cognitivo y lo psicosocial, considerando la existencia de elementos cognitivos que hacen que el individuo se trace objetivos y trabaje en la búsqueda de estos.

Katz (1992) propone un modelo psicosocial y cuestiona los modelos desde los que se pretende explicar el espiritu emprendedor, este autor identifica tres problemas en estos modelos:

- Presentan dificultad para explicar todos los grupos, etnias, familia, marginalidad social

- Tratan el proceso de decisión de manera individual, sin mostrar como la historia familiar o social influyen en las decisiones; y por último,

- No informan acerca de los fracasos del individuo.

Las teorías de la psicología social sugieren que la motivación empresarial está determinada por la creencia de las personas en su capacidad de ejercer control sobre el comportamiento empresarial y tener éxito (creencias de autoeficacia o factibilidad) en un contexto social dado. Si el interés es lo suficientemente fuerte, las personas tendrán la intención de crear una nueva empresa. Esta intención puede o no conducir a la acción, en otras palabras, el emprendimiento sólo tiene lugar cuando es deseable y factible para las personas en su contexto sociopsicológico (Turkina \& Thi, 2015)

\subsubsection{El enfoque Basado en las Oportunidades}

Aquí el emprendimiento es desarrollado gracias a las oportunidades que ofrece el entorno, y es el individuo quien a través de su capacidad, detecta y aprovecha dichas oportunidades. Es así como Shane \& Venkataraman (2000) proponen un enfoque basado en las oportunidades, afirmando que para que haya emprendimiento tienen que existir oportunidades empresariales.

Para Reynolds et al. (2004) el emprendimiento es visto como el descubrimiento de oportunidades y posteriormente la creación de nuevas unidades productivas. Del mismo modo Cuervo, Ribeiro \& Roig (2007) definen al emprendedor como aquel individuo que detecta oportunidades de negocio, las cuales explota a través de la creación de nuevas empresas y ratifican la importancia del espiritu empresarial en el proceso económico.

\subsubsection{El enfoque Etológico}

Estudia al emprendedor desde lo biológico, analizando sus comportamientos como resultado de su interacción con el entorno a través del proceso evolutivo.

El significado y el alcance de la etología según $\mathrm{Pe}$ rinat (1980) es el estudio biológico del comportamiento. Y ese actuar del individuo o del emprendedor es estudiado a partir de cuatro ángulos:

- ¿Qué estímulos (internos o externos) han desencadenado el comportamiento?

- ¿A qué propende este comportamiento, o sea, cuál es su función?

- ¿Cómo este comportamiento ha surgido dentro del conjunto de pautas que caracterizan a un grupo de individuos?

- ¿Qué valor adaptativo o de supervivencia tiene este comportamiento? (Tinbergen, 1963, pp. 410-423)

Para Marulanda, Correa \& Mejía (2009) este factor puede explicar el bajo impacto de los emprendimientos por necesidad en la economía, debido a que estos se orientan básicamente a satisfacer las necesidades de supervivencia del individuo. En este sentido, se plantea que muchas de las iniciativas de 
la generación de ideas para el mejoramiento de la economía mundial, no salieron precisamente de la búsqueda de riqueza, si no de los comportamientos innatos de la condición de racionalidad del ser humano, de las necesidades de supervivencia, dependencia y adaptación a su hábitat y sentimiento por defender su propiedad, aunque algunos psicólogos sustentan que las razones provienen más de la satisfacción de las necesidades propias del ser: individualismo, acción en función de un control voluntario, superación de su dependencia y control por parte del entorno (Marulanda, Correa \& Mejía, 2009).

Retomando el elemento biológico, frente a la pregunta sobre si el emprendedor nace o se hace, la literatura plantea que es una mezcla de ambos. El poseer características internas de personalidad como la motivación, el logro, la independencia y la creatividad, entre otros, no son condiciones únicas para que una persona desarrolle enfoques emprendedores en su entorno, economía o país. Es precisamente la mezcla de factores culturales, familiares, laborales, los que interactúan para potenciar dichas habilidades. Se requiere de ambos para que se dé el carácter emprendedor (Marulanda, Correa \& Mejía, 2009).

\subsection{Factores determinantes en la sostenibilidad de los emprendimientos}

Los factores que pueden afectar la sostenibilidad de los emprendimientos se clasificaron en: factores internos, relacionados con las características de la empresa; factores del emprendedor, referentes a sus habilidades y rasgos, y factores del entorno, relacionados con el ecosistema en el que interactúa la empresa. Algunas observaciones encontradas en la literatura son:

\subsubsection{Factores Internos}

- Tamaño. Este factor genera opiniones contradictorias entre los autores. En cuanto al tamaño inicial de la empresa, medida por el total de activos, (Arias, Jung \& Peña, 2007) plantean que este no incide en la supervivencia del emprendimiento, conclusión que es producto del análisis de las empresas creadas en España en el periodo 1995 - 2002 donde se observó por qué unas empresas duran más que otras y cuáles eran los factores que incidian en dicha duración, para lo cual uso un modelo de regresión lineal y otro de regresión logística.

Martínez (2006, p.113) quien analizó la probabilidad de supervivencia de las empresas industriales constituidas entre 1994 y 2003 en el área metropolitana de Cali - Colombia, a través de un modelo de duración, concluyó que "las empresas entrantes presentan mayores dificultades para consolidarse en los mercados, si su tamaño inicial es pequeño".

Taymaz \& Köksal (2006, p.92) quienes analizaron la supervivencia de las empresas turcas del sector textil a traves de un modelo simple de dos periodos, argumentan que "si la star-up es grande tendrá mayor probabilidad de sobrevivir, pero el impacto del tamaño sobre la probabilidad de salida se reduce con el tiempo".

Igualmente, Parra (2011) quien realizó un análisis de quiebra para las PYMES de Bogotá que se crearon entre 2004 y 2005, con un modelo Probit, concluyó que el tamaño óptimo con el cual debe iniciar un emprendimiento es de la pyme, ya que dicho tamaño minimiza la posibilidad de que esta cierre prematuramente o antes de los primeros cinco años.

Mas-Verdú, Ribeiro-Soriano \& Roig-Tierno (2015) plantean que el tamaño de las empresas es un factor que debe combinarse con otras características a fin de garantizar la supervivencia empresarial, tales como, la combinación entre tecnología y gran tamaño, la combinación del tamaño con el sector (manufacturas) y el apoyo de incubadoras.

Entonces, se podría pensar que el tamaño ideal sería aquel que permita cubrir la demanda actual, y que, para la futura, se hagan inversiones escalonadas, ya que, para una empresa naciente, iniciar con un tamaño que cubra ambas demandas, implicaría mayores costos, producto de la capacidad ociosa instalada, que serían difíciles de cubrir.

- Crecimiento. Según Phillips \& Kirchhoff (1989) un volumen de operación más elevados (mayor producción y ventas), además de garantizar un mejor posicionamiento en el mercado, genera una 
mayor probabilidad de sostenibilidad. Igualmente, Arias et al. (2007, p.15) indican que "empresas con mayor generación de empleo y a su vez mayores inversiones, posibilitan una sostenibilidad más prolongada, acompañada de niveles de productividad y rentabilidad".

Ha (2012) encuentra que existe una relación no lineal entre el crecimiento de las firmas, medido en términos de empleos y activos, y la supervivencia; el cual es mayor en las primeras etapas de la firma donde ésta tiende a crecer a tasas más altas, y luego se va reduciendo conforme el crecimiento de las empresas se va estabilizando.

- Endeudamiento. Valores extremos en los niveles de deuda, ya sean estos muy elevados o minúsculos, exponen los emprendimientos al cierre de estos. En este sentido Parra (2011) sugiere que el nivel de deuda medio y medio-alto, reduce la probabilidad de insostenibilidad de la empresa.

Arias et al. (2007) indican que la forma como se apalanque la empresa, es decir, la procedencia de las fuentes de financiaiòn, es un factor clave en la permanencia del emprendimiento en el mercado. En este sentido señalan que los emprendedores que cuenta con un mayor musculo financiero al inicio de sus operaciones, prologan la existencia de la empresa. De igual forma, hacen hincapié en los plazos de los apalancamientos, afirmando que una mayor concentración de fuente de corto plazo, precipita el cierre de las empresas.

Como complemento a lo anterior, las investigaciones previas sobre el tema plantean que la imposibilidad de los empresarios minoritarios para acceder a créditos y la carencia de capital propio suficiente para el inicio de la empresa, contribuye a las mayores tasas de fracaso en las empresas hispanas y se convierte en una barrera para el tamaño y el alcance de los negocios (Canedo, Stone, Black \& Lukaszewski, 2014).

No solo la deuda usada para comprar activos fijos como muebles o para capital de trabajo, influye sobre la sostenibilidad de los emprendimientos. También la deuda hipotecaria disminuye la sostenibilidad y la probabilidad de que los individuos creen una empresa, y esta es aún menor cuando el emprendimiento se presenta en sectores con mayor riesgo, como lo platean Bracke, Hilber \& Silva (2015), quienes utilizaron una base de datos de panel de individuos en Inglaterra entre 1991 y 2008.

\subsubsection{Factores del Emprendedor}

- Edad. Fertala (2008) analizó la supervivencia de 110.250 empresas liquidadas y en operación, constituidas en Alemania durante 1997 y 2004 por extranjeros o nativos, a través del modelo de riesgos de Gompertz y Makehan, y concluyó que emprendedores que se encontraban entre los 18 y 25 y entre los 36 y 45 años, obtenían mejores resultados en el corto como en el largo plazo. El último lugar lo preservó para aquellos que estaban entre los 26 y 35 años.

- Experiencia. Este factor es considerado por diversos autores como una de las variables que aporta positivamente a la sostenibilidad de los emprendimientos (Rocha, Carneiro \& Amorim 2015; Jung \& Peña 2004), sustentando que la inexperiencia de los emprendedores es una de las causas que puede llevarlos al fracaso aún en condiciones de mercado favorables, ya que puede conducirlos a tomar decisiones equivocadas en cuanto a los niveles de endeudamiento, crecimiento y recuperación de la inversión,

En este mismo sentido, Ronstadt (1984), propone que la experiencia contempla diferentes categorías: i) experiencia educativa, se refiere al proceso formativo del emprendedor y puede incidir positiva o negativamente en la sostenibilidad de la empresa, dependiendo del modelo educativo en el que fue formada la persona, ii) experiencia técnica, iii) experiencia sectorial, iv) experiencia de gestión $y, v$ ) experiencia emprendedora, estas cuatro categorías inciden positivamente en la sostenibilidad de las empresas. Así mismo, algunos autores como Bird (1989) plantean que la experiencia de gestión es una de las principales causas de fracaso de las nuevas empresas.

- Formación. El capital humano del emprendedor, entendido como la cantidad y calidad de conoci- 
miento adquirido, el grado de aprendizaje y de productividad es catalogado como uno de los factores más relevantes en la sostenibilidad de una nueva empresa. Por tanto, las probabilidades de lograr el éxito empresarial serán mayores en la medida en que su capital humano sea más abundante y productivo (Aranguren, Larrea \& Peña, 1999).

La nula asistencia a cursos de formación, la carencia de un título universitario y el hecho de no haber desempeñado previamente un cargo directivo o de gestión contribuyen de manera negativa al mayor logro de los objetivos iniciales marcados por el emprendedor (Aranguren, Larrea \& Peña, 1999).

A pesar de lo anterior, en la literatura, se encuentran posiciones contradictorias frente a la relación entre el nivel de formación de los emprendedores y la actividad emprendedora, es así como algunos estudios establecen una relación negativa entre el nivel educativo y el espíritu empresarial, lo que sugiere que la actividad de crear empresa es más común en aquellas personas que no tienen un alto nivel educativo, lo anterior debido a que la educación superior puede generar mejores opciones externas en el empleo remunerado haciendo menos probable la consideración del emprendimiento como una opción de carrera deseable. Sin embargo, algunos autores plantean lo contrario, es decir que existe una relación positiva entre los niveles más altos de educación y la probabilidad de crear un negocio (Noguera, Âlvarez, Merigó \& Urbano, 2015).

Otros factores relacionados con el emprendedor que se encuentran en las investigaciones están relacionados con el grado de satisfacción de las personas con respecto a su empleo y sus niveles de ingresos. En la medida en que se genere insatisfacción con el empleo actual las personas pueden considerar iniciar su propio negocio (Noguera, Álvarez, Merigó \& Urbano, 2015).

De igual forma Canedo, et al. (2014) destacan factores como la motivación. Indicando que, las personas están motivadas para convertirse en empresarios porque tienen un deseo de riqueza y altas necesidades de logro o autonomía, además se plantea que la experiencia previa, el nivel educativo, la situación socioeconómica y los valores culturales están relacionadas con la motivación de una persona En este sentido, plantean que las personas con niveles de educación más bajos tienen menos probabilidades de estar motivados para convertirse en empresarios $y$ a menudo son menos exitosos que aquellos con niveles de educación superiores.

\subsubsection{Factores del Entorno}

- Crecimiento económico. Uno de los principales factores que pueden afectar la sostenibilidad de una empresa emprendedora es el desarrollo económico del sector; éste se entiende como la capacidad de crear empresas, generar empleo y riqueza, así como la posibilidad de promover y estabilizar un bienestar económico en el sector, con base en las condiciones previas a la creación de la empresa. Este factor se mide generalmente con el Producto Interno Bruto (PIB). El desarrollo económico de la región afecta considerablemente el posicionamiento y el dinamismo de la compañía, en relación a la oferta y la demanda de bienes. El desarrollo económico de una región se ve perjudicado si se percibe un alto nivel de desempleo y se pronostican pocas posibilidades de supervivencia de las nuevas empresas si esta tasa de desempleo tiende a aumentar (Fertala, 2008)

Groszkowski \& Stryjewski (2015) indican que el espíritu emprendedor se ve afectado por aspectos socioeconómicos y políticos del momento histórico, los cuales pueden influir de manera diferente, bien sea generando efectos benéficos sobre la economía tales como incentivos que promueven la dinámica empresarial y económica o, en su defecto, pueden limitar o detener la creación de nuevas empresas y el avance de las ya existentes. Por lo tanto, la creación de condiciones adecuadas para el desarrollo del espíritu empresarial debería ser una de las prioridades más importantes para el desarrollo regional de las autoridades a cualquier nivel.

La mayoría de los estudios sobre la motivación para crear empresas, se enfocan en los factores políticos y económicos tales como el crecimiento económico y las estructuras de gobierno. Sin embargo, la racionalidad económica dominante, es decir, la falta de opciones alternativas de empleo que impulsa a 
las personas a convertirse en trabajadores independientes, parece proporcionar una buena explicación de la necesidad de emprender, pero, no explica en su totalidad la varianza de la oportunidad empresarial (Turkina \& Thi, 2015).

- Crecimiento del sector. El crecimiento del sector al que pertenece la empresa, también es un factor influyente en la supervivencia empresarial, este concepto se refiere al aumento de la productividad y ganancias de un sector específico, en comparación a periodos anteriores. Las empresas ubicadas en sectores que decrecen, presentan un mayor riesgo de fracaso frente a las empresas ubicadas en sectores de bajo crecimiento y alto crecimiento. (Arias \& Quiroga, 2008). Así mismo, una empresa tiene menos probabilidad de fracaso, si se ubica en alguno de los sectores en los que han ingresado la mayor cantidad de nuevas firmas, ya que, a pesar de una mayor competencia, se impulsa el desarrollo del sector (Parra, 2011).

La entrada de nuevas empresas a un sector aporta nuevas formas de competir, lo cual da lugar a nuevas propuestas de valor, que posibilitan el aumento del consumo. A su vez, el mayor crecimiento del sector atraerá nuevas estrategias empresariales que también quieren tener éxito en este (Arias \& Quiroga, 2008).

- Localización geográfica. Parra (2011) plantea que ninguna localización representa un mayor riesgo de fracaso en los primeros cinco años, sin embargo, las empresas ubicadas en sectores con mayor densidad empresarial, tienen menores posibilidades de supervivencia, debido a la saturación y los mayores niveles de competencia.

Algunos autores proponen que la localización tiene un impacto positivo en la supervivencia de las empresas, generando mayores resultados en los sectores con altos niveles de creación de empresas (Arias \& Quiroga, 2008).

- Aglomeración. La posición frente al efecto positivo que puede tener la aglomeración urbana sobre la supervivencia de una empresa, es apoyada por diversos autores (Tavassoli \& Jienwatcharamongkhol,
2016; Fertala, 2008). Dicha aglomeración se entiende como la cantidad de habitantes de la zona, el ordenamiento del conjunto urbano y la distribución entre el núcleo y los suburbios. Inicialmente se dedujo que es sano para una empresa situarse en una región que tenga, aparte de los nativos, personas originarias de otro lugar, ya que esto equilibra un poco el mercado al tener diversidad de usuarios y genera una disminución en la tasa de riesgo para las nuevas empresas.

Es así como, la probabilidad de supervivencia es mayor para las empresas que se establecen en mercados más concentrados, por tanto, las empresas que entran a sectores industriales donde el nivel de concentración es alto o moderado, tienen un riesgo de salida menor de aquellas que hicieron su entrada en mercados económicos con niveles de concentración bajos (Martínez, 2006).

En la literatura se han analizado otros factores referentes al entorno como el reconocimiento de oportunidades, entendido como la oportunidad de generar valor económico que no ha sido explotado previamente por otros. Una oportunidad en el entorno tiene tres características, la rentabilidad, la novedad y la aceptación moral o conveniencia deseada. En este sentido, las personas que tienen acceso considerable a información sobre los mercados y clientes tienen más probabilidades de generar nuevas oportunidades; así mismo, las personas que son altamente creativas o tienen altas habilidades cognitivas están más alerta a las nuevas oportunidades que se generan en el entorno (Canedo, et al., 2014).

Para terminar esta sección, Noguera, et al. (2015) concluyen que los factores informales como la carrera emprendedora y las redes de relaciones tienen una mayor influencia en la actividad emprendedora por encima de los factores formales como la educación, el contexto familiar y el diferencial en los niveles de ingresos

\subsection{Modelo de Cox}

Los modelos de duración son métodos más utilizados en el análisis de supervivencia empresarial, ya que permiten capturar la temporalidad y la varia- 
ción de las circunstancias en el tiempo, mientras que modelos como las regresiónes probabilísticas tipo logit/probit o análisis discriminante, son de naturaleza estática (Arias \& Quiroga, 2008). Asimismo, los modelos tradicionales tienden a subvaluar la información contenida en las observaciones censuradas, ya que se considera la probabilidad de fallo después del período de estudio, lo que puede sesgar los resultados obtenidos (Allison, 1984).

El modelo de riesgos proporcionales de Cox, es un modelo de duración semiparamétrico que permite estimar la supervivencia por medio del método de regresión múltiple.

Según Flores-Luna et al. (2000), el modelo de Cox indica cómo cambia la función de riesgo de base (empresas con el nivel de covariables igual a cero) respecto de las demás (empresas con el nivel de covariables diferente de cero). Este cambio lo recoge el parámetro asociado a cada variable en el modelo, y se interpreta como el cambio esperado en el coeficiente de riesgos entre un individuo en la población de base y uno fuera de ella.

\section{Metodología}

Se aplicó una metodología cuantitativa con un enfoque descriptivo y explicativo, para lo cual se realizó un análisis estadístico a través de un método semiparamétrico (Modelo Cox) en dos momentos: en el primero se incluyeron las covariables asociadas a la supervivencia de manera individual, y en el segundo se incluyeron los efectos cruzados entre las covariables.

Para la estimación del modelo se usó el software IBM SPSS 21. En el método se pretende ajustar el siguiente modelo de regresión que estima la tasa de riesgo en el momento " $\mathrm{t}$ ", como se presenta en la ecuación (1), (Cox, 1972):

$$
\lambda(t ; \boldsymbol{z})=e^{(\boldsymbol{z} \boldsymbol{\beta})} \lambda_{0}(t)
$$

Donde es la tasa instantánea de riesgo en el momento " $\mathrm{t}$ "; $\mathrm{Z}$ es el vector de covariables incluidas en el modelo como factores explicativos de la supervivencia; $\beta$ es el vector de parámetros a estimar asociados a cada uno de los cofactores, y es la tasa de riesgo del modelo de base, es decir, la tasa de riesgo cuando todas las covariables son cero. La expresión es la función paramétrica denominada puntaje de riesgo.

Tomando logaritmo natural a la anterior ecuación se obtiene para la i-ésima ecuación el resultado de las ecuaciones (2) - (4):

$$
\begin{aligned}
& n\left[\lambda_{i}(t)\right]=\ln \left[e^{\left(\beta_{1} X_{i 1}+\beta_{2} X_{i 2}+\cdots+k X_{i k}\right)} * \lambda_{0}(t)\right] \\
& \ln \left[\lambda_{i}(t)\right]=\ln \left[\lambda_{0}(t)\right]+\ln \left[e^{\left(\beta_{1} X_{i 1}+\beta_{2} X_{i 2}+\cdots+k X_{i k}\right)}\right] \\
& n\left[\lambda_{i}(t)\right]=\alpha(t)+\beta_{1} X_{i 1}+\beta_{2} X_{i 2}+\cdots+\beta_{k} X_{i k}
\end{aligned}
$$

Donde $\alpha(t)=\ln \left[\lambda_{0}(t)\right]$

Los parámetros $\beta_{K} \forall K=1,2, \ldots, k$ no varían en el tiempo, razón por la cual a este modelo también se le conoce como modelo de riesgos proporcionales de Cox, debido a que el cociente entre el riesgo para dos empresas con el mismo vector de covariables $\mathrm{Zi}$ es constante en el tiempo (Arias \& Quiroga, 2008).

Este modelo supone que el riesgo de fracaso es constante durante todo el período de estudio, por lo que es preciso validar que los datos muestren evidencia a favor de éste. Al respecto, se realizaron regresiones auxiliares de Cox para cada una de las covariables en el modelo, introduciendo como variable dependiente la variable duración y como covariables cada una de las regresoras (una por cada modelo auxiliar), y un efecto de interacción entre el tiempo y la regresora incluida.

Para la investigación se consultaron los siguientes grupos poblacionales: emprendimientos activos empresas con operación entre 2 y 5 años - y emprendimientos clausurados, en la Subregión del Bajo Cauca, Antioquia.

La muestra estuvo conformada por 101 emprendimientos, 73 activos y 28 inactivos. El método de muestreo se basó en contactos -Network Sam- 
pling-, la selección se realizó a través de las bases de datos suministradas por las Entidades de Fomento.

La información se recolectó a través de encuestas y cuestionarios que indagaban sobre información relacionada con tres ítems: información general del Emprendimiento, características de los emprendedores, y el emprendimiento y el análisis de los factores que influyen en la sostenibilidad de los emprendimientos según la mirada de los emprendedores y las entidades de fomento al emprendimiento.

\section{Resultados}

De acuerdo a la metodología descrita, los resultados se presentan en dos momentos, así:

Una modelación de las variables de manera individual, cuyos resultados son pertinentes cruzar con los resultados obtenidos en el artículo denominado Sostenibilidad de los emprendimientos: Un análisis de los factores determinantes (Sepúlveda \& Reina, 2015), en la cual se usó un modelo no paramétrico (Kaplan \& Meier, 1958), ya que la presente investigación es continuidad de esta. Bajo ese lente, se encontraron variables o factores comunes en ambos modelos, que inciden en la sostenibilidad de las empresas, tales como:

- La formulación del plan de negocios

- La forma de constitución

- El endeudamiento financiero

- La diversificación de la oferta de productos

- La ubicación de la empresa

En este sentido, las variables (a) formulación de plan de negocios y (d) diversificación de la oferta, aportan positivamente a la sostenibilidad del emprendimiento, mientras que la (b) forma constitución de la empresa, específicamente constituirse como S.A.S bajo el modelo de Cox, afecta negativamente la supervivencia, así como (c) el endeudamiento financiero y (e) la ubicación de la empresa, cuando está en sector residencial.

Otras variables que resultaron significativas, desde el modelo de Cox fueron Edad mayor de 30 años y Emprendimiento serial, las cuales afectan de forma positiva y negativa, respectivamente, la sostenibilidad de los emprendimientos. ${ }^{3}$

Tabla 1. Estimaciones Regresión de Cox

\begin{tabular}{|c|c|c|c|}
\hline Omnibus test: & Chi-Cuadrado $=49.578$ & \multicolumn{2}{|c|}{ significancia $=0.000$} \\
\hline Dep Var= Duración (meses) & No. Observaciones & \multicolumn{2}{|c|}{101} \\
\hline Covariables & $\beta$ & Sig. & $\operatorname{Exp}(\beta)$ \\
\hline Ubicación residencial & $2.440^{\star * *}$ & 0.001 & 11.475 \\
\hline Emprendedor desde la Infancia & -0.649 & 0.260 & 0.523 \\
\hline Realización Plan de negocios & $-1.727^{\star \star}$ & 0.023 & 0.178 \\
\hline Diversificación de la oferta & $-0.911 *$ & 0.084 & 0.402 \\
\hline Experiencia & -0.094 & 0.883 & 0.911 \\
\hline Endeudamiento financiero & $1.015^{\star}$ & 0.060 & 2.759 \\
\hline Trayectoria Familiar en emprendimiento & -0.225 & 0.724 & 0.798 \\
\hline Edad entre 20 y 30 & -0.548 & 0.474 & 0.578 \\
\hline
\end{tabular}

3 Se recomienda a los lectores revisar el articulo Sostenibilidad de los emprendimientos: Un análisis de los factores determinantes (Sepúlveda \& Reina, 2015), con el fin de realizar un paralelo entre los dos artículos. 


\begin{tabular}{|c|c|c|c|}
\hline Omnibus test: & Chi-Cuadrado $=49.578$ & \multicolumn{2}{|c|}{ significancia $=0.000$} \\
\hline Dep Var= Duración (meses) & No. Observaciones & \multicolumn{2}{|c|}{101} \\
\hline Covariables & $\beta$ & Sig. & $\operatorname{Exp}(\beta)$ \\
\hline Edad Mayor que 30 & $-1.421^{*}$ & 0.066 & 0.242 \\
\hline Género & -0.478 & 0.349 & 0.620 \\
\hline Emprendimiento Serial & $1.350^{\star}$ & 0.062 & 3.857 \\
\hline Variables Macro & 0.369 & 0.544 & 1.446 \\
\hline Municipio & 0.709 & 0.422 & 2.032 \\
\hline Apoyo entidades de fomento & -0.543 & 0.344 & 0.581 \\
\hline Forma Jurídica SAS & $1.978^{\star \star}$ & 0.040 & 7.225 \\
\hline Forma Jurídica Persona Natural & 0.650 & 0.550 & 1.916 \\
\hline Forma Jurídica Sin Registrar & 1.491 & 0.106 & 4.441 \\
\hline Tamaño Famiempresa & -0.883 & 0.217 & 0.413 \\
\hline Formación Intermedia & 1.214 & 0.142 & 3.368 \\
\hline Formación Superior & 0.526 & 0.485 & 1.693 \\
\hline
\end{tabular}

Nota: los tres asteriscos indican niveles de significancia individual al $1 \%$, dos asteriscos, al $5 \%$ y con un asterisco al $10 \%$. Los coeficientes sin asteriscos resultaron no significativos a ninguno de los valores estándar anteriores. Las variables resaltadas son las que presentan significancia en el modelo de Kaplan como en el de Cox

Fuente: Elaboración de los autores con datos del software SPSS 21

Dado que los valores ajustados por el modelo de regresión de Cox se deben comparar con los del modelo de base, en el que las covariables son todas iguales a cero, a continuación, se presenta dicho modelo, en el cual se encuentran las empresas con las siguientes características:

- Están ubicadas en la zona comercial, industrial o rural.

- El emprendedor no considera que tenía aptitudes de emprendedor desde la infancia.

- No se realizó un plan de negocios.

- No se diversificó la oferta de productos.

- El emprendedor tenía endeudamiento financiero.

- El emprendedor no tenía una trayectoria familiar en emprendimiento.

- El emprendedor tiene menos de 20 años de edad.

- El emprendedor es del género femenino.

- El emprendedor no es serial.

- El emprendedor no consideró alguna variable macro al momento de crear la empresa.
- La empresa está ubicada en el municipio de Caucasia.

- El emprendedor no recibió apoyo de alguna entidad.

- La forma jurídica de la empresa es diferente a una SAS, persona natural o sin registrar.

- El tamaño de la empresa es familiar o pequeña.

- La formación educativa del emprendedor es básica (primaria o bachillerato).

En la Tabla 1, la columna de los coeficientes estimados $(\beta)$ indica en qué dirección afecta cada factor al riesgo de fracaso o subsistencia. Es decir, si dicho coeficiente es positivo, el factor bajo estudio implica un mayor riesgo de fracaso (menor probabilidad de subsistencia), mientras que si es negativo indica un menor riesgo de fracaso (mayor probabilidad de subsistencia). Por su parte, la columna "exp ( $\beta$ )" es la exponencial del valor obtenido de cada parámetro, por ejemplo, para el caso de la variable "ubicación residencial", su coeficiente estimado fue de 2,440 , cuyo exponencial es este resultado indica el número de veces que el factor bajo estudio aumenta 
la tasa de riesgo de fracaso en relación al grupo del modelo de base descrito anteriormente. Entonces, la tasa de riesgo de las empresas ubicadas en zonas residenciales es 11,475 veces mayor en relación a una empresa que se encuentre en otra zona (comercial, industrial o rural).

Según lo anterior, la Tabla 1 indica que las variables que promueven una mayor supervivencia son: elaboración de un plan de negocios, diversificación de la oferta y edad mayor a 30, ya que son las que tiene un coeficiente $\beta$ negativo. Mientras que las variables: ubicación residencial, endeudamiento financiero, emprendimiento serial, y forma jurídica SAS, presentan una relación negativa con la supervivencia.

Entonces, la probabilidad de fracaso de una empresa que realizó plan de negocios, es de 1,822 veces menor que una del modelo de base.

Así mismo, las empresas donde la oferta se diversificó, son 1.598 veces menos probables de fracasar, y las empresas que tenían endeudamiento financiero en sus inicios, son 2,759 veces más proclives al fracaso. Los resultados también indican que empresas cuyos empresarios tienen más de 30 años de edad son 1,758 veces menos probables de fracasar, lo cual es evidencia a favor de que personas con mayor experiencia (la edad es una proxi de esta variable) pueden tener empresas más exitosas.

Con respecto a la variable "emprendimiento serial", según la tabla 1, una empresa creada por un individuo con experiencia en la creación de empresas, tiene 3,853 más probabilidad de fracasar, resultado que es contra intuitivo en relación con lo encontrado en otros estudios (Cooper, Woo, \& Dunkelberg, 1989; Stuart \& Albetti, 1990), ya que se espera que la experiencia con los emprendimientos sea un factor que permita lograr un mayor éxito en futuras empresas. Sin embargo, Boden \& Nucci (2000), tampoco encuentran una relación sistemática de la experiencia en el manejo de empresas del emprendedor y la supervivencia de la empresa, aunque si logran establecer que las empresas cuyos dueños tienen mayor escolaridad y más de 10 años de experiencia de trabajo sobreviven más, lo cual es contrario a lo encontrado en este estudio, donde la formación resultó ser una variable no significativa.

Por último, las empresas constituidas bajo la forma jurídica SAS son 7.225 veces más riesgosas que aquellas que se constituyeron bajo otra forma jurídica.

En el otro momento se incluyeron los efectos de interacción entre las diversas variables (experiencia y haber considerado más de una variable macroeconómica al iniciar el emprendimiento; tener actitudes de emprendimiento desde la infancia y familiares emprendedores; la experiencia y el emprendimiento serial; tamaño del emprendimiento y la formación del emprendedor, entre otras $\left.{ }^{4}\right)$, y al final de este ejercicio, sólo se encontró un efecto de interacción estadísticamente significativo entre las variables Experiencia y haber considerado más de una variable macroeconómica al iniciar el emprendimiento, y que no alteraba sustancialmente los resultados del modelo obtenido en el primer momento.

La variable de interacción de la regresora con el tiempo no fue significativa en ninguno de los modelos auxiliares, por lo que se concluye que ninguna de las covariables incluidas en el modelo de regresión de Cox presentado tiene efectos temporales.

En este segundo momento, las variables adicionales a las del primer momento, que influyen estadísticamente en la supervivencia de las empresas estudiadas son: Genero, Variables macroeconómicas, formación intermedia y la relación entre experiencia y variables macroeconómicas, como se puede apreciar en la tabla 2, la cual presenta los resultados de la estimación del modelo de regresión de Cox con el análisis de interacción entre las variables

4 En total se ajustaron diez modelos con efectos de interacción. 
Tabla 2. Resultado modelo de regresión de Cox con efectos de interacción

\begin{tabular}{|c|c|c|}
\hline Omnibus test: & Chi-Cuadrado $=45.460$ & significancia $=0.002$ \\
\hline \multirow[t]{2}{*}{ Dep Var= Duración (meses) } & No. Observaciones & 101 \\
\hline & B & $\operatorname{Exp}(B)$ \\
\hline Ubicación residencial & $2.577^{\star \star \star}$ & 13.160 \\
\hline Emprendedor desde la Infancia & -0.772 & 0.462 \\
\hline Realización Plan de negocios & $-1.811^{\star \star}$ & 0.164 \\
\hline Diversificación de la oferta & $-0.970^{*}$ & 0.379 \\
\hline Experiencia & 0.944 & 2.569 \\
\hline Endeudamiento financiero & $1.030^{*}$ & 2.802 \\
\hline Trayectoria Familiar & -0.436 & 0.647 \\
\hline Edad entre 20 y 30 & -0.270 & 0.763 \\
\hline Edad Mayor que 30 & $-1.380^{*}$ & 0.252 \\
\hline Género & $-0.963^{*}$ & 0.382 \\
\hline Emprendimiento Serial & $1.359 *$ & 3.892 \\
\hline Variables Macro & $1.421^{*}$ & 4.140 \\
\hline Municipio & 0.731 & 2.077 \\
\hline Apoyo & -0.774 & 0.461 \\
\hline Forma Jurídica SAS & $1.969^{* *}$ & 7.162 \\
\hline Forma Jurídica Persona Natural & 0.712 & 2.037 \\
\hline Forma Jurídica Sin Registrar & 1.359 & 3.894 \\
\hline Tamaño Famiempresa & -0.766 & 0.465 \\
\hline Formación Intermedia & $1.636^{\star}$ & 5.133 \\
\hline Formación Superior & 0.614 & 1.847 \\
\hline experiencia*var_macro & $-2.305^{\star}$ & 0.100 \\
\hline
\end{tabular}

Nota: los tres asteriscos indican niveles de significancia individual al $1 \%$. dos asteriscos. al $5 \%$ y con un asterisco al $10 \%$. Los coeficientes sin asteriscos resultaron no significativos a ninguno de los valores estándar anteriores.

Fuente: elaboración de los autores, con datos del software SPSS 21.

Como se aprecia en la tabla 2, las magnitudes de los coeficientes de las variables que resultaron significativas en el modelo presentado en la tabla 1 no cambian significativamente respecto al modelo inicial, incluso su signo se conserva. Adicionalmente, en este nuevo modelo se logran rescatar otras variables para el análisis, como: género, variables macro económicas y formación intermedia, las cuales resultaron ser significativas en la supervivencia de las empresas.
Con respecto al género, las empresas creadas por emprendedores hombres tienen un riesgo menor de fracaso, pues la propensión a este es sólo de 0.382 en relación a las creadas por mujeres. Este resultado debe mirarse con cautela, pues la significancia estadística de esta variable se encuentra en el límite (9.6\%).

De manera similar, las empresas creadas por emprendedores que consideraron más de una variable macroeconómica tienden a ser más vulnerables al 
fracaso, ya que tienden a tener una tasa de fracaso 4.1 veces superior que aquellas en las cuales sus emprendedores solo consideraron una o ninguna variable macroeconómica. Este resultado no es tan sorprendente ya que la mayoría de las empresas dentro de la muestra son pequeñas empresas, que suelen surgir como una necesidad de auto empleo en lugar de una oportunidad de negocio, por lo que considerar variables macroeconómicas no es uno de los determinantes a la hora de crear la empresa. Nuevamente, esta variable se encuentra en el límite de la significancia (8.8\%).

Adicionalmente, los resultados indican que, si el emprendedor tiene un nivel de formación intermedia, la empresa será más propensa al fracaso, unas 5.13 veces más que una cuyo emprendedor solo tenga estudios de primaria. Esto puede deberse a que, emprendedores con mayores niveles de formación pueden tener puede obtener más fácilmente un empleo y así, dejar de lado su proyecto empresarial. Mientras que, para los emprendedores con bajo niveles de educación, la mejor opción es seguir continuar con su proyecto de emprendimiento.

Finalmente, la interacción entre las variables experiencia y variable macro indican que, si una persona tiene experiencia y además consideró más de una variable macroeconómica al iniciar su empresa, entonces tendrá una mayor tasa de supervivencia. Este resultado lleva a pensar que la experiencia en un emprendedor le permite aprovechar e interpretar mejor la información de las variables macroeconómicas en aras de potenciar la supervivencia de las empresas.

\section{Conclusiones}

Las variables presentes en ambos momentos de la modelación, a la hora de explicar la supervivencia de los emprendimientos, tales como elaboración de un plan de negocios, diversificación de la oferta y el endeudamiento financiero, tienen un efecto común en la mayoría de los escenarios de las empresas nacientes, ya que:

- Todo emprendimiento debería ser desarrollado a partir de investigaciones objetivas, que, aunque no son garantía de éxito, cuantifican la necesidad real del bien o servicio a generar y por ende permiten establecer la probabilidad de éxito que tendrá el emprendimiento, con lo que se constituyen en una carta de navegación que reduce el riesgo de fracaso, toda vez que sean formulados y evaluados objetivamente.

- Dado que los emprendimientos en su creación son tan frágiles debido a sus bajos niveles de operación, altos niveles de endeudamiento los expone a niveles de riesgo, en ocasiones, insostenibles. En este sentido el apalancamiento financiero se puede convertir en una dificultad que, en lugar de generar valor, lo destruye, generando una contribución financiera negativa, de tal forma que es más conveniente garantizar la inyección de los recursos necesarios con dinero del emprendedor o de fuentes menos cotosas.

- Un crecimiento bien planeado y estructurado tanto en la diversificación de la oferta como en nuevas unidades estratégicas de negocios, que conlleve a un mayor número y mejores propuestas de valor para los clientes, seguramente generará un incremento del valor agregado para la empresa y un mayor sustento en el tiempo, para lo cual se requiere que toda inversión marginal genere una rentabilidad superior al costo incurrido en la misma, y que un incremento en ventas este acompañado de un menor esfuerzo operativo para generarlo.

El hecho de que la forma de constitución de la empresa sea significativa y que aporte negativamente en la sostenibilidad si es una S.A.S, se sustenta cuando se habla de empresas en etapa temprana, para las cuales, dados sus bajos volúmenes de operación, cubrir las erogaciones que implican las formas de constitución diferentes a las personas naturales, es complicado.

Dado que la variable, poseer características de emprendedor desde la infancia, no fue significativa, se podría intuir, que la teoría "biologicista" del emprendimiento, que indica que los emprendedores nacen, no es del todo sostenible, ya que muchos de 
esos rasgos se pueden adquirir en la escuela, tal y como lo propone Wompner (2008) en el Enfoque de los Rasgos de Personalidad.

Con respecto a la educación, cuando se estudia a los emprendedores más reconocidos en el mundo, se encuentra que muchos de ellos han abandonado las aulas de clase prematuramente. Lo anterior obliga a reflexionar sobre la pertinencia de los modelos educativos actuales, los cuales no promueven la creatividad y abordan la educación como un fin y no como un medio, hecho que puede explicar porque un emprendimiento donde el emprendedor presenta una formación intermedia, tenga más posibilidades de desaparecer que aquel que no cuenta con ella, ya que este último no ha pasado por un proceso que castre esa imaginación o creatividad. Adicionalmente, resulta importante observar como mayores oportunidades de empleabilidad y mejores niveles salariales, lo cual se obtiene con un nivel de educación mayor, cambian las preferencias de los individuos a la hora de generar o no su propio empleo.

La no significancia de la experiencia como variable individual, se corrobora cuando se identifica que muchos de los emprendedores, no cuentan con más experiencia que la obtenida en aquellos emprendimientos que marcaron su camino al éxito. Lo anterior coincide con la posición de Ronstadt (1984) y Bird (1989) en relación a la experiencia educativa, y la faceta o experiencia de gestión, respectivamente.

En este mismo sentido, el hecho de que la variable emprendimiento serial afecte negativamente la supervivencia de la empresa, corrobora lo señalado en el párrafo anterior, ya que haber creado emprendimientos previamente, puede generar un sesgo en el emprendedor que lo lleve a asumir posturas viciadas, toda vez que las experiencias de los anteriores emprendimientos no sean debidamente aprovechadas.

En relación con la edad ideal para ser emprendedor, a pesar de que desde el modelo semiparametrico, tienen mayor probabilidad de éxito los mayores de 30 años, sería arriesgado tomar este resultado como condición para la supervivencia de las empresas, ya que, si bien los emprendedores jóvenes adolecen de la experiencia y la intuición de un emprendedor mayor, estos cuentan con la energía, el entusiasmo y la determinación, necesarias en cualquier emprendedor de éxito. Por lo anterior, resulta más lógico presumir que el resultado encontrado en el modelo no paramétrico, donde la edad no es significativa en la supervivencia (Sepúlveda \& Reina, 2015), es más acertado.

El hecho de que la variable "recibir apoyo de entidades de fomento al emprendimiento" no fuera significativa en ningún modelo, indica que las entidades de fomento y el gobierno deben hacer más énfasis en las fases de aceleración, incubación y postincubación de los emprendimientos, las cuales actualmente no son muy atendidas así como realizar un filtro más exhaustivo en la selección de los emprendimientos a apalancar, de tal forma que se concentre en aquellos que presenten mayores niveles de innovación y mejores propuestas de valor para los clientes, y así optimizar los recursos que se destinan al emprendimiento.

Pese a todo lo anterior, es necesario no solo analizar las variables de manera independiente, ya que, al cruzarlas, las sinergias que se pueden presentar entre estas, cambian la incidencia de las mismas en la supervivencia de los emprendimientos, toda vez que, en presencia de algunas habilidades, en este caso la experiencia, se puede potenciar el provecho que se puede obtener de otras variables.

La presente investigación aporta un análisis cuantitativo de los factores que afectan la sostenibilidad de las empresas, indicando precisamente cuantas veces una característica del emprendimiento, del emprendedor o del entorno, aporta negativa o positivamente en la sostenibilidad de la empresa, frente a otra que no posee tal característica, lo cual se traduce en una herramienta que puede ser usada por todas las fuerzas vivas comprometidas con el tema del emprendimiento.

Posteriores investigaciones deben trabajar con muestras donde los diversos sectores de la economía estén igualmente desarrollados y los tamaños de empresas sean más diversos, así como los tipos de emprendedores en cuanto a la formación. 


\section{Referencias}

Allison, P. (1984) Event history analysis. Londres: Sage Publications.

Aranguren, M. J., Larrea, M. \& Peña, I. (1999). Incubadoras: ¿Supervivencia y Crecimiento de Nuevas Empresas?. Encuentro de Economía Aplicada, Reus. 1-27.

Arias, A., Jung, A. \& Peña, I. (2007). Factores asociados al cese de actividades de nuevas firmas españolas. Cuaderno de Economía, Uruguay, Departamento de Economía, Facultad de Ciencias Empresariales, Universidad Católica del Uruguay II (2), 7-21

Arias, A. \& Quiroga, R. F. (2008). Cese de actividades de las pymes en el área metropolitana de Cali (2000-2004): un análisis de supervivencia empresarial. Cuaderno Administración Bogotá, XXI (35), 249-277.

Baumol, W. J. (1996). Entrepreneurship : Productive, Unproductive and Destructive. Journal of Business Venturing, 11 (1): 3-22.

Bird, B. (1989). Entrepreneurial Behavior. Scott, Foresman and Company. En: Glenview (IL). BIS.

Boden, R. J. \& Nucci, A. R. (2000) On the survival prospects of men's and women's new business ventures. Journal of Business Venturing, XV (4), 347-362. Disponible: http:// doi.org/10.1016/S0883-9026(98)00004-4.

Bracke, P., Hilber, C. \& Silva, O. (2015). Mortgage debt and entrepreneurship (Bank of England working papers). En: Bank of England. Retrieved from, https://ideas.repec.org/p/boe/ boeewp/0560.html.

Cámara de Comercio de Medellín para Antioquia. (2013). Permanencia Empresarial en Antioquia en el período 2000-2012. Revista Antioqueña de Economía y Desarrollo RAED, ed. (6): $45-85$

Campbell, C. A. (1992). A decision theory model for entrepreneurial acts. Entrepreneurship: Theory and Practice. p. 21-28.

Canedo, J., Stone, D., Black, S. \& Lukaszewski, K. (2014). Individual factors affecting entrepreneurship in Hispanics. Journal of Managerial Psychology, 29 (6): 755-772.

Casson, M. (1983). The Entrepreneur- An Economic Theory. Albro Business History Review. 570-572

Cooper, A. C., Woo, C. Y. \& Dunkelberg, W. C. (1989) Entrepreneurship and the initial size of firms. Journal of Business Venturing, IV (5): 317-332, Disponible: http://doi.org/http:// dx.doi.org/10.1016/0883-9026(89)90004-9.

Cox, D. R. (1972). Regression Models and Life-Tables. Journal of the Royal Statistical Society. Series B (Methodological), XXXIV (2), 187-220. Disponible: http://doi.org/10.2307/2985181.

Cuervo, Á., Ribeiro, D. \& Roig, S. (2007). Entrepreneurship: Conceptos, Teoría y Perspectiva. Revista de la Universidad de Valencia.

Fertala, N. (2008). The shadow of death: do regional differences matter for firm survival across native and immigrant entrepreneurs?. Empirica, XXXV (1), 59-80.

Flores-Luna, C, M., Muñoz, S. Z., Salazar-Martínez, E. \& LazcanoPonce, E. (2000). Análisis de supervivencia. Aplicación en una muestra de mujeres con cáncer cervical en México. Salud pública de México, 42 (3), 242-251.

Gartner, W. B. (1988). "Who is an Entrepreneur?" Is the Wrong Question. Educational Foundation, 11-32.

Groszkowski, T. \& Stryjewski, T. (2015). The Econometric Analysis of Entrepreneurship Determinants in Polish Voivodeships in the Years 2004-2013. Dynamic Econometric Models. 15, 157-165.

Ha, N. (2012). The effect of growth on firm survival in vietnam. Depocen Working Paper Series, 18.

Iturriaga, M., Baniandrés, J. \& Eizaguirre, A. (2016) Del emprendimiento individual a la capacidad emprendedora de nuestras organizaciones: un análisis de los factores que la definen. Boletin de estudios económicos. LXXI (217): 49-69

Jung, A. \& Peña, I. (2004). Factores asociados al fracaso de nuevas empresas. En: III Conferencia Internacional de Investigadores en Emprendedurismo de América Latina, Rio de Janeiro, 56-68.

Kaplan, E, \& Meier, P. (1958). Nonparametric estimation from incomplete observations. Journal of the American Statistical Association, LIII (282), 457-481.

Katz, J. A. (1992). A Psychosocial Cognitive Model of Employment Status Choice. Entrepreneurship: Theory y Practice, 29-37.

Lau, T. \& Chan, K. F. (1994). The Incident Method - An Alternative Way of Studying Entrepreneurial Behaviour. Irish Business and Administrative Research. 15: 48

Martínez, A. F. (2006). Determinantes de la supervivencia de empresas industriales en el área metropolitana de Cali 19942003. Revista Sociedad y Economía. (11), 112-144.

Marulanda, J. A., Correa, G. \& Mejía, L. F. (2009). Emprendimiento: Visiones desde las teorías del comportamiento humano. Revista EAN, (66): 153-168.

Mas-Verdú, F., Ribeiro-Soriano, D. \& Roig-Tierno, N. (2015). Firm survival: The role of incubators and business characteristics. Journal of Business Research, 68(4), 793-796. http://doi. org/DOI: $10.1016 /$ j.jbusres.2014.11.

Moriano, J. A., Trejo, E. \& Palací, F. J. (2001). El Perfil Psicosocial del Emprendedor: Un estudio desde la perspectiva de los valores. Revista de Psicología Social, (16): 229-242.

Noguera, M., Alvarez, C., Merigó, J. \& Urbano, D. (2015). Determinants of female entrepreneurship in Spain: an institutional approach. Computational and Mathematical Organization Theory. 21 (4): 341-355.

Parra, J. F. (2011). Determinantes de la probabilidad de cierre de nuevas empresas en Bogotá. Revista Facultad de Ciencias Económicas: Investigación y Reflexión, XIX (1), 27-53.

Pereira, F. \& Medina, L. (2012). Global Entrepreneurship Monitor-GEM-Antioquia 2012-2013. Colombia: Ediciones Sello Javeriano.

Perinat, A. (1980). Contribuciones de la Etología al Estudio del Desarrollo Humano y Socialización. El Basilisco, (11): 27-34.

Phillips, B. \& Kirchhoff, B. (1989). Formation, Growth and Survival; Small Firm Dynamics in the U.S. Economy. Small Business Economics, I (1), 65-74. 
Rajasekaran (2013). Sustainable Entrepreneurship: Past Researches and Future Directions. Journal of Entrepreneurship and Management, 2 (1): 20-27.

Reynolds, P. D., Carter, N. M., Gartner, W. B. \& Greene, P. G. (2004). The Prevalence of Nascent Entrepreneurs in the United States: Evidence from the Panel Study of Entrepreneurial Dynamics. Small Business Economics, (23), 263-284.

Rocha, V., Carneiro, A. \& Amorim Varum, C. (2015). Serial entrepreneurship, learning by doing and self-selection. International Journal of Industrial Organization, 40: 91-106. http://doi.org/10.1016/j.ijindorg.2015.04.001

Rodriguez, A. (2009). Nuevas perspectivas para entender el emprendimiento empresarial. Pensamiento y Gestión, (26): 94-119.

Ronstadt, R. S. (1984). Entrepreneurship: Text, Cases and Mites. Lord Publishing, Dover (MA).

Schaltegger, S. \& Wagner, M. (2011). Sustainable Entrepreneurship and Sustainability Innovation: Categories and Interactions. Business Strategy and the Environment Business, (20): 222-237.

Sepulveda, C. \& Reina, W. (2015). Sostenibilidad de los emprendimientos: Un análisis de los factores determinantes. Revista Venezolana de Gerenecia (23), 33-49

Shane, S. \& Venkataraman, S. (2000). The Promise Of Entrepreneurship As A Field Of Research. Academy of Management Review, (25), 217-227.
Simon, V. M., Revuelto L.T. \& Medina, A. F. (2012). La influencia de la formación, la experiencia y la motivación para emprender en la supervivencia de las empresas de nueva creación. Estudios gerenciales, XXVIII, (Edición especial), 237-262.

Stuart, R. W. \& Abetti, P. A. (1990). Impact of entrepreneurial and management experience on early performance. Journal of Business Venturing, V (3), 151-162, Disponible: http:// doi.org/http://dx.doi.org/10.1016/0883-9026(90)90029-S.

Tavassoli, S. \& Jienwatcharamongkhol, V. (2016). Survival of Entrepreneurial Firms : The Role of Agglomeration. Externalities Papers in Innovation Studies. CIRCLE, (October)

Taymaz, E; Köksal, M. (2006). El espíritu emprendedor, el tamaño de lanzamiento y la supervivencia de los pequeños empresarios. En: Ekonomiaz: Revista vasca de economia, (62): 70-99.

Tinbergen, N. (1963). On Aims and Methods of Ethology. Department of Zoology. Revista University of Oxford, 20: 410-433.

Turkina, E. \& Thi, M. T. (2015). Socio-psychological determinants of opportunity entrepreneurship. International Entrepreneurship and Management Journal, 11 (1): 213-238

Wompner, F. (2008). Educación superior para el emprendimiento. Expansiva. Serie indagación 19.

Yeasmin, N. (2016). The Determinants of Sustainable Entrepreneurship of Immigrants in Lapland: En Analysis of Theoretical Factors. Entrepreneurial Business and Economics Review, 4(1): 129-159. 